\title{
Adenopathy and fibrosis in thoracic sarcoidosis occurring concurrently-A rare presentation
}

\author{
Rajul Rastogi*, Prabhat Kumar Bhagat, Yuktika Gupta, Pankaj Kumar Das, Mohini Choudhary, Sagar Parashar, and Vijai Pratap \\ Teerthanker Mahaveer Medical College and Research Center, Moradabad, UP, India
}

\begin{abstract}
Sarcoidosis is a common multisystem disorder affecting the thoracic region. A well-defined, clinical staging system already exists for thoracic sarcoidosis. Rarely, combinations of signs / symptoms or imaging features may occur that may challenge the staging system as seen in our case and may force us to realize that our understanding of the disease pattern is still in the stage of evolution. In this article, we are describing a rare combination of adenopathy with pulmonary fibrosis (first and final stage features) in thoracic sarcoidosis at presentation.
\end{abstract}

\section{Introduction}

Sarcoidosis is a noninfectious, granulomatous disease affecting multiple systems of the human body. Respiratory system or thoracic cavity is invariably affected in the disease. A clinical staging system exists based on the imaging features and determines the prognosis of the patient. In this article, we present an interesting case of thoracic sarcoidosis where the imaging features of stage I \& stage IV were detected concurrently (with latter also at uncommon location) at the time of presentation.

\section{Case report}

A 30-years old male presented with few months' onset of slight dyspnea on exertion. Clinical examination revealed fine crepts at bilateral pulmonary bases; more marked on right side. History of obvious fever and cough was denied. Laboratory examination including serum ACE levels was unremarkable. Radiograph of the chest in PA projection was advised which revealed doubtful right hilar adenopathy with infiltrative lesions in right pulmonary basal region. Contrastenhanced computed tomography (CECT) of the chest was then advised for further evaluation.

CECT thorax in mediastinal window setting revealed nearsymmetric, homogeneous, bilateral, discrete, hilar adenopathy along with subcarinal adenopathy without obvious internal calcification or rim enhancement (Figure 1 \& 2). Lung window settings revealed presence of interstitial septal thickening with few cyst formation involving basal segments of right lower lobe associated with subpleural fibrotic changes, pleural tagging and slight loss of volume of right lower lobe. Small area of fibroatelectatic changes in left basal lung was also noted (Figure $1 \& 2$ ). Based on these findings, diagnosis of thoracic sarcoidosis with stage I (adenopathy) and stage IV (pulmonary fibrotic lesions) findings were suggested.

Transbronchial biopsy of mediastinal \& hilar adenopathy revealed noncaseating granulomas confirming the diagnosis. Patient was then advised short-duration steroid therapy after completion of which patient remained asymptomatic for six months before being lost in follow-up.

\section{Discussion}

Thoracic sarcoidosis, a multisystem, noninfectious, granulomatous, inflammatory disease characterized histologically by formation of noncaseating granuloma in affected tissues, is being increasing reported from the Indian subcontinent. Imaging plays an important role in the staging of the disease which is as follows:

Stage 0: Normal chest findings



Figure 1. A-D. Transaxial CECT chest images in mediastinal-window settings (A,B) show subcarinal \& bilateral hilar adenopathy (white arrows) and lung-window settings (C,D) show fibrotic changes in bilateral basal lungs (black arrows); more marked on right side.

Correspondence to: Rajul Rastogi, Assistant Professor, Department of Radiodiagnosis, Teerthanker Mahaveer Medical College and Research Center, Moradabad, Uttar Pradesh, India-244001, Tel: 919319942162; E-mail: eesharastogi@gmail.com

Keywords: sarcoidosi, adenopathy, pulmonary, fibrosis

Received: August 25, 2016; Accepted: September 27, 2016; Published: September 30, 2016 


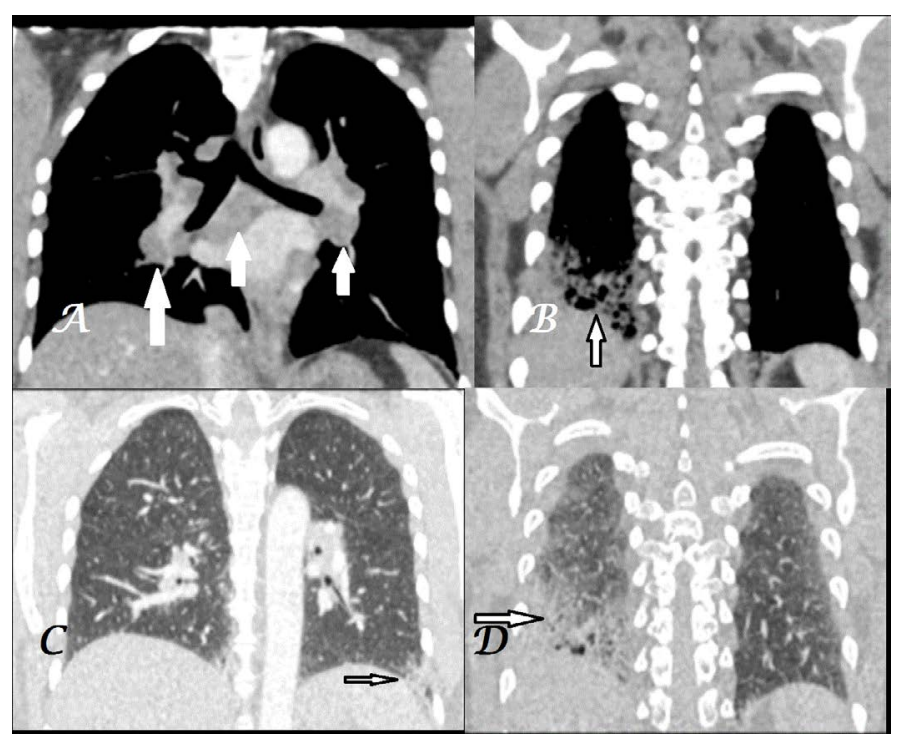

Figure 2. A-D. Coronal CECT chest images in mediastinal-window settings (A) show subcarinal \& bilateral hilar adenopathy (white arrows) and mediastinal (B) with lungwindow settings (C,D) show fibrotic changes in bilateral basal lungs (black arrows); more marked on

Stage 1: Bilateral hilar and / or mediastinal adenopathy

Stage 2: Adenopathy with pulmonary parenchymal infiltrates

Stage: 3: Pulmonary parenchymal infiltrates only

Stage IV: Pulmonary fibrosis

The above-described staging system not only helps in determining mode of management (NSAID vs steroid therapy) but also helps in monitoring treatment and predicting prognosis [1]. Advanced stage of the disease is associated with poorer prognosis and morbidity \& mortality is primarily related to development of pulmonary hypertension \& respiratory failure secondary to pulmonary fibrosis [2].

Enlarged hilar (usually symmetrical) and mediastinal nodes, first stage of disease is the commonest mode of presentation at the times of diagnosis with less than one-fifth showing nodal calcification $[3,4]$.

Pulmonary parenchymal interstitial infiltrative lesions (often symmetrical) occurring primarily in the central zones of the upper lobes may occur with or without adenopathy comprising of Stage II \& Stage III disease respectively [3]. Though sarcoidosis is primarily an interstitial disease yet airspace consolidation with air-bronchogram is not infrequently seen. Progression of disease leads to irreversible pulmonary fibrosis with loss of normal volume \& pulmonary parenchymal architecture as well as development of honeycombing, bronchiectasis, bullae \& rarely cavitation in the affected regions of lung [5].

Thus our case is unique as both stage I (adenopathy) and stage IV (pulmonary fibrosis) findings were seen concurrently at the time of presentation with latter being at uncommon location. This case teaches us that the use of staging system in thoracic sarcoidosis cannot be overemphasized and more number of similar cases may make the changes in the staging system imperative.

\section{Conclusion}

Though present system of staging of thoracic sarcoidosis helps in management, monitoring of treatment and predicting prognosis yet deviations in presentation of disease (concurrent existence of stage I \& IV manifestations) may make the clinical task difficult.

\section{References}

1. Criado E, Sanchez M, Ramifrez J, Arguis P, De Caralt TM, et al. (2010) Pulmonary sarcoidosis: Typical and atypical manifestations at high-resolution $\mathrm{CT}$ with pathologic correlation. Radiographics 30: 1567-1586.

2. Nardi A, Brillet PY, Letoumelin P, Girard F, Brauner M, et al. (2011) Stage IV sarcoidosis: comparison of survival with the general population and causes of death. Eur Respir J 38: 1368-1373.[Crossref]

3. Deepak D, Shah A (2001) Thoracic sarcoidosis. The spectrum of roentgenologic appearing. Indian J Radiol Imaging 11: 191-198.

4. Lynch JP, Kazerooni EA, Gay SE (1997) Pulmonary sarcoidosis. Clin Chest Med 18 755-785.[Crossref]

5. Baughman RP, Winget DB, Bowen EH, Lower EE (1997) Predicting respiratory failure in sarcoidosis patients. Sarcoidosis Vasc Diffuse Lung Dis 14: 154-158.

Copyright: $@ 2016$ Rastogi R. This is an open-access article distributed under the terms of the Creative Commons Attribution License, which permits unrestricted use, distribution, and reproduction in any medium, provided the original author and source are credited. 